\title{
Les travaux de restauration de la grotte du château de La Bâtie d'Urfé à Saint-Étienne-le-Molard (Loire)
}

Restoration work in the grotto of the château de La Bâtie d'Urfé in SaintÉtienne-le-Molard (Loire)

\section{Jean-François Grange-Chavanis}

\section{(2) OpenEdition}

Journals

Édition électronique

URL : http://journals.openedition.org/artefact/5282

DOI : 10.4000/artefact.5282

ISSN : 2606-9245

Éditeur :

Association Artefact. Techniques histoire et sciences humaines, Presses universitaires du Midi

Édition imprimée

Date de publication : 15 juillet 2020

Pagination : $39-51$

ISBN : 978-2-8107-0691-4

ISSN : 2273-0753

\section{Référence électronique}

Jean-François Grange-Chavanis, «Les travaux de restauration de la grotte du château de La Bâtie d'Urfé à Saint-Étienne-le-Molard (Loire) », Artefact [En ligne], 12 | 2020, mis en ligne le 21 décembre 2020, consulté le 23 décembre 2020. URL : http://journals.openedition.org/artefact/5282 ; DOI : https://doi.org/10.4000/artefact.5282

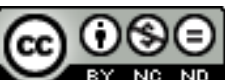

Artefact, Techniques, histoire et sciences humaines est mise à disposition selon les termes de la Licence Creative Commons Attribution - Pas d'Utilisation Commerciale - Pas de Modification 4.0 International. 


\section{Les travaux de restauration de la grotte du château de La Bâtie d'Urfé à Saint-Étienne-le-Molard (Loire)}

Jean-François Grange-Chavanis

\section{Résumé}

La grotte de rocailles conçue et réalisée au milieu du $x v{ }^{e}$ siècle au château de La Bâtie d'Urfé est la plus authentique et la mieux conservée de toutes celles qui ont été construites avant elle en France et en Italie dans le souvenir ravivé de l'Antiquité. Une restauration très complète menée à bien en 2008 a redonné leur lustre à tous les éléments qui la composent. Pavage, murs ornés de personnages de rocailles variées et de coquillages, plafond de bois décoré de sables colorés, grilles de fer forgé, statues de marbre, forment un espace d'une profonde originalité qui sert d'atrium à la chapelle du château où s'unissent amour humain et amour divin dans un extrême raffinement.

\section{Mots-clés}

Claude d'Urfé, Renaissance, rocailles, coquillages, restauration

95 Jean-François Grange-Chavanis, « Les travaux de restauration de la grotte du château de La Bâtie d'Urfé à Saint-Étienne-le-Molard (Loire) », Artefact, 12, 2020, p. 39-51. 


\section{Restoration work in the grotto of the château de La Bâtie d'Urfé in Saint- Etienne-le-Molard (Loire)}

\section{Abstract}

The grotto of rockeries designed and built in the middle of the $16^{\text {th }}$ century in the chateau of La Bâtie d'Urfé is undoubtedly the oldest and most authentic of all those built at the time of the Renaissance in France and Italy. A very complete restoration carried out in 2008 has given back their lustre to all the elements that make it up. Paving, walls adorned with various stone figures and shells, a wooden ceiling decorated with coloured sands, wrought iron grills, marble statues, form a space of profound originality that serves as an atrium for the chapel where human and divine love are united in extreme refinement.

\section{Keywords}

40 Claude d'Urfé, Renaissance, rockeries, shells, restoration 


\section{Introduction}

Le château de la Bâtie d'Urfé est situé dans la plaine de la Loire à l'ouest de Lyon sur la route de l'Auvergne au centre de l'ancienne province du Forez (Fig. III, cahier couleur). D'origine médiévale, une simple maison-forte a été métamorphosée au milieu du XVI ${ }^{\mathrm{e}}$ siècle en un château moderne par Claude d'Urfé (1501-1558). Celui-ci fit du fief de ses ancêtres la matérialisation inachevée d'un rêve de perfection mêlant de façon très originale traditions françaises et nouveautés italiennes. Homme profondément religieux, cultivé, polyglotte, humaniste, Claude d'Urfé est nommé par François Ir $^{\text {er }} 1546$ ambassadeur auprès du Concile de Trente, puis auprès du Pape Paul III Farnèse par Henri II en 1548. Déjà familiarisé avec la péninsule par sa participation aux guerres d'Italie, il passe alors son temps en voyages incessants de Trente à Ferrare, Bologne, Rome, Gênes probablement et à la cour à Fontainebleau et Paris où il termine sa carrière comme gouverneur des Enfants de France et membre du Conseil de régence, passant sans doute trop rapidement dans sa demeure forézienne ${ }^{1}$. C'est dire qu'il est amené à côtoyer l'aristocratie civile et religieuse de son temps, les artistes et les intellectuels qui gravitent autour d'elle dans l'entourage immédiat de la famille royale française. La Bâtie est le reflet fidèle de cette activité débordante, sans que jamais les simples effets de mode prennent le pas sur la culture, la spiritualité, la fidélité à la foi catholique et à l'amour conjugal. C'est ainsi que cette maison concentre en peu d'espace les pièces de fonction habituelles d'une demeure aristocratique, une chapelle introduite par une grotte artificielle et un jardin clos très élaboré.

\section{"La plus belle du royaume "}

Cette grotte, réalisée en peu d'années aux alentours de 1550 est aujourd'hui la plus authentique et la mieux conservée de ces nombreuses cavernes imaginaires dont a raffolé l'élite européenne de l'époque ${ }^{2}$. Le plan général de la Bâtie n'est en rien celui des châteaux habituels de son époque : pas de grand escalier, pas de trace apparente d'une galerie intérieure, apanage de toutes les demeures de même importance, localisation hypothétique de la

\footnotetext{
1. Guichard, 1990.

2. Poulain 1990.
} 
bibliothèque, hiérarchie aléatoire des étages. Il semble avoir été le fruit de décisions contradictoires ou de repentirs, mais seuls nous restent à peu près intacts les deux éléments qui semblent le reflet le plus exact des volontés de leur concepteur : la chapelle et cette grotte qui lui sert d'atrium ${ }^{3}$. C'est là que se concentre l'univers de Claude d'Urfé, c'est là que l'on passe du paganisme, de l'ignorance à la connaissance, à la science, à l'amour humain et à l'amour divin unis dans la chapelle. Si cette dernière a été dépecée, il est heureusement possible de restituer en totalité le décor qui l'ornait dont une partie est encore en place, l'autre dispersée à travers le monde mais bien connue (Fig. 1).

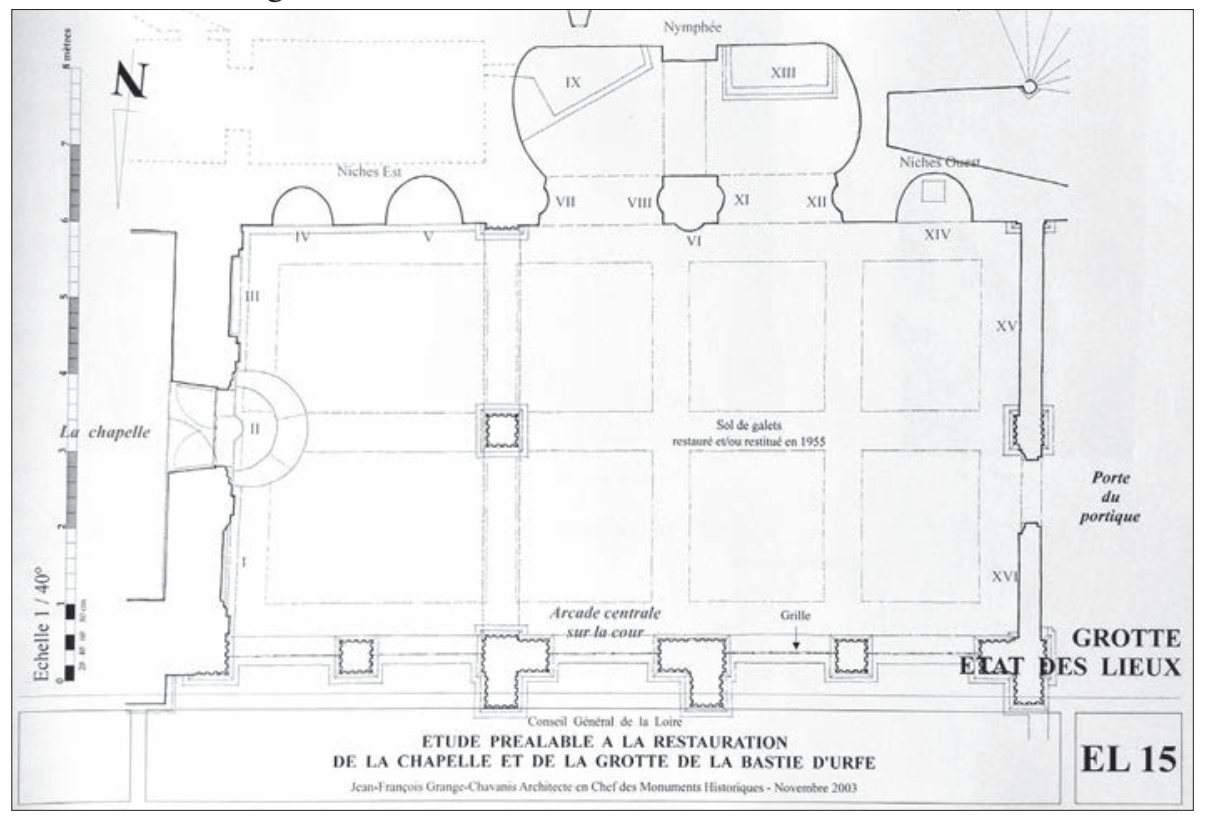

Fig. 1. - Grotte de La Bâtie d'Urfé, état des lieux en 2003 avant restauration

\section{Plan Jean-François Grange-Chavanis}

La grotte, quant à elle, est pratiquement complète : ne manquent à son décor que les statues, sans doute de marbre, qui compléteraient dans leurs niches vides une statue de grande taille, identifiée comme un Vertumne et attribuée à Montorsoli (vers 1507-1563) ${ }^{4}$, et les restes d'une nymphe alanguie sur un dauphin encore en place. La Bâtie d'Urfé est restée

3. Bugini, 2019.

4. Malgouyres 2007, p. 18 et fig. 4 et 5. 
pratiquement inconnue du monde de l'histoire de l'art jusqu'à la fin du $\mathrm{XIX}^{\mathrm{e}}$ siècle, ce qui l'a paradoxalement sauvée de modifications incessantes qui l'auraient dénaturée. Depuis la mort de Claude d'Urfé en 1558, aucun travail de modernisation d'importance n'y a été réalisé et le domaine, tombé en léthargie dès la disparition de son concepteur, n'a fait l'objet d'aucun relevé, d'aucune description précise à l'exception de maigres jalons très espacés dans le temps :

- un dessin réalisé en 1611 par le père jésuite Étienne Martellange qui n’est qu'une vue extérieure très partielle mais précieuse par sa minutie

- un plan terrier de 1804 qui montre l'environnement du château dans un état peut-être inchangé depuis sa conception, mais ne révèle, comme le précédent naturellement, rien des dispositions intérieures ${ }^{6}$;

- un état des lieux de 1778 précis mais trop lacunaire là encore sur l'état intérieur ${ }^{7}$, qui ne décrit pas davantage la grotte.

Néanmoins, deux témoignages anciens mentionnent la grotte : d'abord celui d'Anne d'Urfé, petit-fils de Claude, dans une description du château rédigée vers 1606 : " Il y a aussy une crotte [sic], où il y a plusieurs belles et grandes estatues de marbre rapportées d'Italie, laquelle estoit estimée la plus belle du royaume, au temps qu'elle fut faicte ${ }^{8} »$. Ensuite, celui de Jacques Fodéré qui mentionne également la statuaire mais aussi le décor de rocailles « fait de petites pierres si industrieusement appliquées en figures qu'ils font merveilleusement bien » et les jeux d'eaux?

Le château a été vendu à la suite de la triste déchéance de la famille d'Urfé au XVIII ${ }^{\mathrm{e}}$ siècle. Les ventes se succèdent alors au siècle suivant sans que, par miracle, ni la chapelle ni la grotte n'aient été vidées de leurs œuvres d'art, contrairement au reste de la maison et aux jardins. À la fin du siècle, les choses se gâtent. En 1872, une nouvelle vente à un marchand de biens, prélude à un démantèlement complet et presque à une démolition, aboutit en un premier temps au démontage du pavement, des boiseries, des panneaux de marqueterie qu'elles enchâssent, des toiles, de l'autel, des vitraux de la chapelle, décor dont ne subsiste alors en place que ce qui ne

5. Martellange, 1611.

6. "Plan géométral... », ca 1804.

7. "Visite sommaire prisée des réparations à faire aux batimens du château, moulin et domaine de la terre et seigneurie de la Bâtie ", $1^{\text {er }}$ juillet 1778, Archives départementales de La Loire, ancien minutier de Bourdelon, [document disparu] ; cité par Bugini, 2019, p. 27-28, n. 6.

8. A. d'Urfé, vers 1606, dans Bernard, 1839, p. 450 (cité par Poulain, 1990, p. 111).

9. Fodéré, 1619, p. 984 ; cité par Bernard, 1839, p. 474 et par Poulain, 1990, p. 111. 
peut pas être démonté, à savoir la voûte en stuc, les pilastres de pierre et les arcs qui scandent l'espace. Tous ces éléments sont très officiellement vendus et leur trace suivie, d'antiquaires en acquéreurs, de collectionneurs privés en musées, jusqu’à leur retour partiel et leur remise en place dans les années 1950.

La grotte, quant à elle, reste inchangée, à l'exception de la porte d'entrée sur la cour et des statues qui l'ornent, elles aussi vendues, dont l'une reviendra après quelques voyages et l'autre restée dans les lieux, sa mutilation en ayant peut-être empêché la commercialisation. Deux événements heureux viennent alors à la fin $\mathrm{du}_{\mathrm{XIX}}{ }^{\mathrm{e}}$ siècle et au début $\mathrm{du} \mathrm{Xx}^{\mathrm{e}}$ siècle interrompre le cycle des malheurs de la Bâtie : en 1886, le comte Georges de Soultrait écrit l'histoire du château illustrée de photographies de Félix Thiollier qui fixent de façon définitive l'aspect des lieux dans tous leurs détails avant leur démantèlement ${ }^{10}$. Puis, en 1909, la Diana, société historique sise à Montbrison, fondée en 1862, emblématique des activités de recherche et de publication de grande qualité de l'époque, rachète le château et ses abords immédiats pour les sauver de la démolition. Cet achat entraîne le classement au titre des monuments historiques en 1912 du château qui se trouve ainsi à l'abri des risques qu'il encourait jusque-là.

\section{Conserver et restaurer}

La seconde partie du Xx ${ }^{\mathrm{e}}$ siècle voit, dans les années 1950-1960, des travaux de restauration et d'aménagement se dérouler dans le but d'ouvrir dignement la Bâtie au public, travaux dont la poursuite devient urgente dès les années 1990, date où le conseil général de la Loire (aujourd’hui conseil départemental), prend le relais de la Diana dans le cadre d'un bail emphytéotique pour assumer le financement, avec l'aide de l'État, puis l'animation du domaine et son exploitation.

L'archéologie a été depuis cette période constamment présente à la Bâtie, tant en sous-sol qu'en élévation, des campagnes de fouilles très fructueuses ayant considérablement amélioré la connaissance des bâtiments et des

10. De Soultrait et Thiollier, 1886. 
jardins sous la houlette de Christian le Barrier ${ }^{11}$ et d'Anne Allimant ${ }^{12}$ au fur et à mesure du lancement des projets. Une étude générale de restauration et de mise en valeur est alors établie pour établir les priorités d'intervention et leur coût ${ }^{13}$. Après les toitures, les façades, les jardins, une étude préalable à la restauration de la chapelle et de la grotte (Fig. 1) est menée à bien en $2004^{14}$. Après approbation, cette étude aboutit en 2007 à un projet complet sur les bases duquel les travaux de restauration de la grotte sont menés à bien jusqu'à leur achèvement en 2010. La restauration de la chapelle, quant à elle, est restée pour l'instant à l'état de projet en raison des questions complexes que posent les principes de sa réhabilitation et de l'évocation des décors dont le retour n'est pas envisageable : boiseries et marqueteries au Metropolitan Museum de New York, carreaux du pavement répartis entre plusieurs collections. L'extrême qualité de ces œuvres d'art rend l'idée d'une copie difficilement envisageable, plusieurs tentatives s'étant révélées peu concluantes et l'authenticité reste pour ces chefs-d'œuvre un critère primordial.

Les travaux de la grotte n'auraient pas pu être menés à bien sans l'établissement d'un état des lieux extrêmement précis fondé sur l'observation de tous les matériaux qui composent cet ensemble unique. Un relevé pierre à pierre des murs a d'abord été réalisé par Anne Carcel, conservateur des Antiquités et objets d'art de la Loire, sous la forme de très beaux dessins en couleurs $^{15}$. En 1992, Robert Bouiller, alors conservateur du musée Alice Taverne d'Ambierle et ancien collaborateur du Service géologique national, assisté de Bernard Guyot, compagnon du devoir, et de Jean-Roch Bouiller, étudiant en histoire de l'art, avait consigné ses « Observations sur les matériaux constituant les rocailles de la nymphée à La Bastie-d'Urfé » dans un rapport essentiel pour la connaissance des pierres et leur éventuelle provenance $^{16}$. Luc Goupil, architecte du patrimoine, compléta ce relevé et ce rapport par la représentation du plafond, du sol, des grilles et de l'ancienne porte d'entrée sur la cour et l'analyse de leur état, précisant ainsi l'étude préalable de 2004 et la transformant en projet architectural et technique.

11. Le Barrier, 2004. L'intervention a eu lieu du 16 février au 10 mars 2004.

12. Anne Allimant a effectué des fouilles archéologiques dans les jardins du château de La Bâtie d'Urfé de 1993 à 1998.

13. Grange-Chavanis, 2019.

14. Grange-Chavanis, 2004.

15. Dessins sur calques, coll. part.

16. Bouiller, 1992. 
Ce document, dont le volet financier fut réalisé par Joël Jermer, économiste vérificateur des monuments historiques, a servi de base à une consultation des artisans et des entreprises habilités à réaliser les travaux décrits dans le cadre de la réglementation des marchés publics. Cette consultation effectuée, les travaux envisagés ont pu se dérouler de janvier à décembre 2008 et ont fait l'objet en 2010 d'un dossier des ouvrages exécutés exposant de façon minutieuse, en particulier pour le plafond et les murs, les travaux réalisés ${ }^{17}$. La restauration du plafond a été confiée à Carlo Usai, chargé à Rome des " Restauro e conservazione di beni culturali ${ }^{18}$ ", celle des rocailles à l'Atelier de Conservation Eschlimann, établi en Alsace et à Paris ${ }^{19}$. Sont aussi intervenus l'entreprise Comte pour la maçonnerie-pierre de taille, l'assainissement et le système d'alimentation en eau, l'entreprise Chantelot pour la menuiserie, l'Atelier Rocle pour la ferronnerie et l'entreprise Bréat pour l'électricité.

\section{Les décors}

La grotte de la Bâtie est unique par sa conception, ses dispositions et la date de sa réalisation approximativement contemporaine de celle de la chapelle puisqu'il faut traverser l'une pour accéder à l'autre. C'est sans doute à partir de 1548 et avant 1558, date de la mort de Claude d'Urfé, que l'aménagement des deux espaces a été mené à bien, les seules certitudes de datation précise concernant la chapelle. La grotte devrait donc être la plus ancienne construction de rocailles, de coquillages, de stuc et de bois conservée en Europe dans un tel état d'authenticité, toutes les autres grottes comparables, en France comme en Italie en particulier ayant disparu, ayant été considérablement modifiées ou ayant été réalisées plus tard. Il semble néanmoins que ce soient les grottes génoises et florentines qui s'avèrent les plus proches par l'esprit et les techniques, bien que l'on ne sache pratiquement rien des équipes de "rocailleurs ", probablement italiennes et itinérantes, qui les auraient mises en œuvre. Leur technicité avait été soulignée par Robert Bouiller :

17. Les archives du chantier de restauration seront déposées à la Médiathèque de l'architecture et du patrimoine (Charenton-le-Pont).

18. Usai, 2008a et 2008b.

19. Szkotnicki, 2008. 
\ک Les rocailleurs ont mis en œuvre : des coquilles actuelles d'origine marine ; des fossiles; des roches ou minéraux des environs; des galets et des minéraux importés; la technique du stuc. Ils ont joué à la fois sur la taille, sur la forme et la faculté d'orienter les fragments lithiques, sur leurs couleurs bien entendu, mais aussi avec l'éclat, souvent amoindri aujourd'hui. La maîtrise ressort de la conjugaison adroite de tous ces effets ${ }^{20}$.

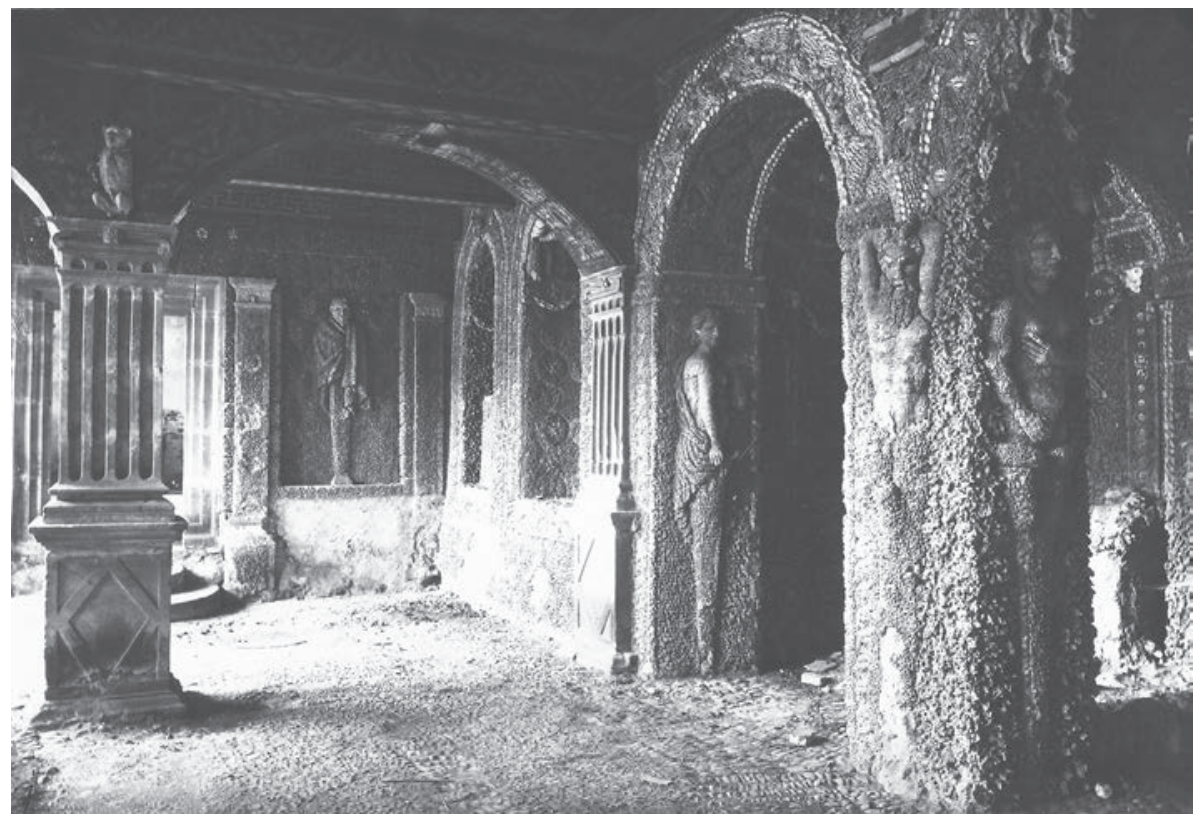

\section{Fig. 2. - Grotte de La Bâtie d'Urfé, photographie de Félix Thiolliez vers 1880}

\section{Coll. part. Jean-François Grange-Chavanis}

Si l'on en juge par les photographies de Félix Thiollier ${ }^{21}$, datées des années 1880 , la grotte ne semble pas avoir été modifiée à cette date depuis sa réalisation (Fig. 2). Puis les travaux des années 1950-1960 montrent que seuls quelques compléments sur les murs de rocailles, sans bouchement systématique des lacunes, ont été réalisés un peu maladroitement car aucune observation exhaustive n'avait eu lieu à cette époque. Le sol en

20. Bouiller, 1992, p. 4.

21. Reproduites dans De Soultrait et Thiollier, 1889 ; des tirages originaux supplémentaires sont également conservés dans une collection privée des descendants de Félix Thiollier. 
revanche avait été refait à neuf du fait de son très mauvais état bien visible sur les photographies du XIX ${ }^{\mathrm{e}}$ siècle. Une partie du pavage original avait été néanmoins conservée, servant de modèle assez respectueusement imité. Ce nouveau sol a donc été conservé lors de la dernière campagne de travaux, simplement supprimé sur une largeur de 20 centimètres au pied des murs pour améliorer l'assainissement et diminuer les remontées d'humidité présentes en périphérie, remontées provoquées à la fois par le regrettable comblement des douves, qui entouraient la plate-forme d'assise du château, et l'utilisation d'un mortier de ciment dans la chape de base du nouveau sol. Seuls les pavages des deux grandes niches du mur sud, qui n'avaient pas été concernés par la campagne 1950-1960, ont fait l'objet d'une restitution fidèle aux résultats des sondages archéologiques réalisés.

Les murs, quant à eux, ont été brossés et nettoyés avec précaution, et aucun élément nouveau n'est venu compléter les rocailles et les coquillages originaux et les interventions très réduites de la précédente campagne. Les lacunes trop voyantes ont été simplement atténuées par un lait de chaux léger de la couleur des pierres existantes, en particulier sur les galets minuscules simulant l'épiderme des personnages. Quelques consolidations ont été effectuées là où cela était nécessaire avec un mortier de chaux dont la composition est la plus proche possible de l'original. Les matériaux employés sont principalement des variétés de rocailles : du granite rouge, du granite gris, du granité sombre, des scories volcaniques, du quartz blanc, du quartz rose, du quartz bréchique ferrugineux, du schiste ardoisier, du grès houiller, du calcaire blanc, du carbonate en rhomboèdres, enfin des galets plats, des galets ovoïdes, du travertin et des fossiles. L'origine locale des roches granitiques et des quartz est probable, mais une grande partie des rocailles était importée ${ }^{22}$. Par ailleurs, la décoration emploie en abondance des coquilles marines, ce qui implique bien évidemment des échanges commerciaux lointains (Fig. IV, cahier couleur).

L'intervention la plus visible a concerné le plafond qui n'avait pas été touché par la campagne des années 1950-1960. Ce plafond est constitué de planches de bois fixées par des clous de fer forgé sur une structure classique qu'on pourrait dire "à la française ». Ces planches sont décorées par divers sables colorés fixés à la colle animale ponctués de coquillages. Les analyses faites sur place et en laboratoire ont montré que ce décor ancien

22. Bouiller, 1992, p. 5-10. 
n'avait jamais été restauré, mais que les lacunes étaient importantes, les coquillages n'étant décelables que par leur trace sur le bois et les couleurs ayant perdu beaucoup de leur vivacité. Contrairement à ce qui a été mis en ouvre sur les murs, il a été décidé de rendre au plafond tout l'éclat qu'il avait perdu avec le temps en complétant les lacunes de sable, ravivant les couleurs et replaçant les coquillages dont la cueillette fut réalisée sur la plage de Tarquinia, près de Rome, non loin sans doute de leur lieu d'origine, sans oublier de distinguer les coques lisses des coques crénelées dont on distinguait parfaitement les traces (Fig. V, cahier couleur). Le blason de la famille d'Urfé, dont le bleu avait pu être évoqué par des perles de lapis-lazuli, a été rétabli avec des matériaux plus contemporains et moins onéreux, seule entorse au parti pris d'authenticité qui a présidé à l'ensemble de la restauration, une surface du plafond ayant d'ailleurs, sur environ $1 \mathrm{~m}^{2}$, été laissée dans son état d'origine.

La grotte est encore équipée d'un système d'arrosage, relativement sommaire mais permettant toutefois de faire fonctionner des jets judicieusement placés dans les deux niches principales du mur sud et sur les personnages qui les ornent. Ces jets sont alimentés par un réseau de tuyaux de plomb sortant d'un réservoir s'écoulant par simple gravité. Ce système a été entretenu en état de marche mais l'humidité qu'il entraîne est assez préjudiciable à la bonne conservation des lieux, et son utilisation ne peut être que sporadique. Les grilles de fer forgé simulant des pampres de vigne, restées en très bon état, ont seulement été complétées de quelques feuilles manquantes redorées à la feuille. Enfin la porte d'entrée sur la cour n'a finalement pas été remise en place en raison des difficultés que représentait l'utilisation de moulages de l'original accompagnée de la copie de certains éléments. Il pourra être remédié à cette situation dans les années à venir.

\section{Conclusion}

La grotte de La Bâtie a ainsi retrouvé presque toute sa splendeur d'origine, sans que soient pour autant résolus tous les mystères de sa conception et de sa réalisation, que les recherches ultérieures aideront, il faut l'espérer, à lever. Parmi les grottes ornementales créées au milieu du XvI siècle, elle occupe une place singulière, à l'écart des grandes métropoles et des résidences royales et princières. Elle n'a pas souffert des destructions, transformations ou même des réaménagements qu'ont subis bien d'autres constructions 
comparables, si bien que son relatif abandon au cours des siècles lui a permis de conserver une grande part d'authenticité. Son architecture, sobre et discrète, et son décor, luxueux et raffiné, allient une grande originalité : vestibule d'une chapelle, qu'elle précède et qu'elle complète, elle témoigne d'un projet ambitieux auquel la figure de Claude d'Urfé, commanditaire et probablement ordonnateur des travaux doit être associée. Conçue durant le concile de Trente auquel il participa, la grotte résulte des échanges foisonnants entre l'Italie et la France.

\section{Sources}

Paris, Bibliothèque nationale de France, département des estampes et de la photographie : Étienne Martellange, "Veüe de la Bastie d'Urfé en Forés ", dessin, 1611, Ub 9a, no 128 ; repr. Grange-Chavanis, 2019, p. 78, fig. 1.

Saint-Étienne-le-Molard, château de La Bâtie d'Urfé : "Plan géométral des domaines de la famille Puy de la Bâtie ", ca 1804 ; repr. Grange-Chavanis, 2019, p. 79, fig. 2.

Fodéré Jacques, Narration historique et topographique des convens de l'ordre S. François, P. Rigaud, Lyon, 1619.

\section{Rapports}

Bouiller Robert (dir.), " Observations sur les matériaux constituant les rocailles de la nymphée à La Bastie-d'Urfé », 1992.

Grange-Chavanis Jean-François, "Château de La Bastie d'Urfé, chapelle et grotte ", Étude préalable à la restauration, Conseil général de la Loire, février 2004.

Le BARrier Christian, "La Bâtie d'Urfé (Loire), chapelle et salle de fraîcheur ", Rapport de diagnostic, Institut national de recherches archéologiques préventives, avril 2004.

Usar Carlo, « Loire, St-Étienne le Molard, Bastie d'Urfé, étude préalable pour la restauration du plafond ", février 2008 ; «Batie d'Urfé, grotta, Indagni propedeutiche al restautato conservativo delle decorazioni del soffitto, eseguite con 'graniglie' policrome incollate su intonaco di supporto », juin 2008.

Szkotnicki Bruno, "Relevé des lacunes " (Loire, Saint-Étienne-le-Molard, Château de la bastie d'Urfé, restauration de la grotte, lot $\mathrm{n}^{\circ} 2$ : restauration des rocailles, entreprise Eschlimann), avril 2008. 


\section{Bibliographie}

BERnARD Auguste, Les d'Urfé, souvenirs historiques et littéraires du Forez au XVI et XVII siècles, Imprimerie royale, Paris, 1839.

Bugini Elena (dir.), Sacellum mirabile, Nouvelles études sur la chapelle de Claude d'Urfé, PUR, Rennes, 2019.

De Soultrait Georges et Thiollier Félix, Le château de la Bastie d'Urfé et ses seigneurs, société de La Diana, Saint-Étienne, 1886.

Grange-Chavanis Jean-François, "Apostilles en marge de la dernière campagne de restauration du château de La Bâtie d'Urfé ", dans Bugini Helena (dir.), Sacellum Mirabile, Nouvelles études sur la chapelle de Claude d'Urfé, PUR, Rennes, 2019, p. 77-86.

Guichard Vincent (dir.), Claude d'Urfé et La Bâtie, L'univers d'un gentilhomme de la Renaissance, Conseil général de la Loire, Montbrison, 1990.

Malgouyres Philippe, "Claude d'Urfé, Montorsoli, Giulio Camillo, Charles Quint et quelques autres ", Bulletin de la Société de l'histoire de l'art français, 2007, p. 9-25.

Poulain Dominique, "La grotte ", dans Guichard Vincent (dir.), Claude d'Urfé et La Bâtie, L'univers d'un gentilhomme de la Renaissance, Conseil général de la Loire, Montbrison, 1990, p. 110-119.

\section{L'auteur}

Architecte DPLG en 1972, lauréat du concours d'Architecte en chef des Monuments historiques en 1981, Jean-François Grange-Chavanis a été chargé de la restauration de monuments historiques classés dans le Puy-de-Dôme, le Cantal, le Gard, la Loire (où est situé le château de la Bâtie d'Urfé), la Savoie, la HauteSavoie, l'Isère et à Saint-Louis-des-Français de Lisbonne (Portugal). Contact : jf. grangechavanis@aeclyon.com 